\title{
BMJ Open Prevalence of pre-diabetes and undiagnosed diabetes in the Mollerussa prospective observational cohort study in a semi-rural area of Catalonia
}

To cite: Falguera $\mathrm{M}$, Vilanova MB, Alcubierre N, et al. Prevalence of prediabetes and undiagnosed diabetes in the Mollerussa prospective observational cohort study in a semi-rural area of Catalonia. BMJ Open 2020;10:e033332. doi:10.1136/ bmjopen-2019-033332

- Prepublication history and additional material for this paper are available online. To view these files, please visit the journal online (http://dx.doi org/10.1136/bmjopen-2019033332).

Received 30 July 2019 Revised 05 December 2019 Accepted 07 January 2020

Check for updates

(c) Author(s) (or their employer(s)) 2020. Re-use permitted under CC BY-NC. No commercial re-use. See rights and permissions. Published by BMJ.

For numbered affiliations see end of article.

Correspondence to Dr Didac Mauricio; didacmauricio@gmail.com

Dr Esmeralda Castelblanco; esmeraldacas@gmail.com

\section{ABSTRACT}

Objectives To assess the prevalence of undiagnosed diabetes and pre-diabetes in the healthy population in the Mollerussa cohort. As a secondary objective, to identify the variables associated with these conditions and to describe the changes in glycaemic status after 1 year of follow-up in subjects with pre-diabetes.

Design Prospective observational cohort study.

Setting General population from a semi-rural area. Participants The study included 583 participants without a diagnosis of diabetes recruited between March 2011 and July 2014.

Results The prevalence of undiagnosed diabetes was $20,3.4 \%(95 \% \mathrm{Cl} 2.6$ to 4.2$)$ and that of pre-diabetes was $229,39.3 \%$ (37.3 to 41.3 ). Among those with prediabetes, $18.3 \%$ had isolated impaired fasting plasma glucose (FPG) (FPG: 100 to $<126 \mathrm{mg} / \mathrm{dL}$ ), $58.1 \%$ had isolated impaired glycated haemoglobin $(\mathrm{HbA} 1 \mathrm{c})(\mathrm{HbA} 1 \mathrm{c}$ 5.7 to $<6.5$ ) and $23.6 \%$ fulfilled both criteria. Follow-up data were available for 166 subjects; $41.6 \%$ (37.8 to 45.4 ) returned to normoglycaemia, $57.6 \%$ (57.8 to 61.4$)$ persisted in pre-diabetes and $0.6 \%$ (0 to 1.2) progressed to diabetes. Individuals with pre-diabetes had worse cardiometabolic risk profiles and sociodemographic features than normoglycaemic subjects. In the logistic regression model, variables significantly associated with pre-diabetes were older age (0R; 95\% Cl) $(1.033 ; 1.011$ to 1.056$)$, higher physical activity $(0.546 ; 0.360$ to 0.827$)$, body mass index $(1.121 ; 1.029$ to 1.222$)$ and a family history of diabetes (1.543; 1.025 to 2.323$)$. The variables significantly associated with glycaemic normalisation were older age (0.948; 0.916 to 0.982$)$ and body mass index (0.779; 0.651 to 0.931$)$.

Conclusions Among adults in our region, the estimated prevalence of undiagnosed diabetes was $3.4 \%$ and that of pre-diabetes was $39.3 \%$. After a 1-year follow-up, a small proportion of subjects $(0.6 \%)$ with pre-diabetes progressed to diabetes, while a high proportion (41.6\%) returned to normoglycaemia. Individuals with pre-diabetes who returned to normoglycaemia were younger and had a lower body mass index.

\section{Strengths and limitations of this study}

- This was a population-based study of a small cohort that included a representative sample of a nonpreviously studied population of a semi-rural area in Catalonia.

- We did not perform an oral glucose tolerance test, which is a common test in most studies but is a time-consuming and expensive procedure.

- The small number of cases of undiagnosed diabetes precluded further statistical analyses on this topic.

\section{BACKGROUND}

Diabetes mellitus, a public health concern with an increasing incidence worldwide, is a great threat to general health and is leading to increased morbidity and mortality. These effects are mainly occurring because diabetes is a disorder of glucose metabolism that affects multiple organ systems and is associated with various microvascular and macrovascular complications and several non-vascular complications. Additionally, a large group of subjects do not fulfil the diabetes criteria but have intermediate glycaemic variables, between normal and diabetes, and are thus classified as having pre-diabetes. One of the most commonly used definitions of prediabetes is that of the 2010 American Diabetes Association (ADA) criteria: ${ }^{2}$ (a) impaired fasting plasma glucose (IFG), defined as fasting plasma glucose (FPG) between 100 and $<126 \mathrm{mg} / \mathrm{dL}$ (5.6 to $5.9 \mathrm{mmol} / \mathrm{L})$; (b) impaired glucose tolerance (IGT), defined as a 2-hour plasma glucose value after a $75 \mathrm{~g}$ oral glucose tolerance test (OGTT) between 140 and $<200 \mathrm{mg} / \mathrm{dL}(7.8$ to $11.0 \mathrm{mmol} / \mathrm{L})$ or (c) glycated haemoglobin (HbAlc) levels between $5.7 \%$ and $<6.5 \%$ (39 to $46 \mathrm{mmol}$ ) mol). 
Pre-diabetes is becoming increasingly important as it represents a high risk of developing type 2 diabetes (T2D) and cardiovascular diseases. ${ }^{23}$ Moreover, individuals with pre-diabetes are phenotypically quite similar to patients with T2D. That is, they tend to be older, with a higher body mass index (BMI) and higher blood pressure than people with normal glucose tolerance; in addition, they tend to have insulin resistance and dyslipidaemia. ${ }^{4}$ Additionally, multiple risk factors, such as family history, gestational diabetes and certain ethnicities as well as combined risk factors such as metabolic syndrome, are known to predispose subjects to a higher risk for pre-diabetes and its progression to T2D. ${ }^{5}$ Based only on IGT, the worldwide prevalence of pre-diabetes among adults has been estimated by the International Diabetes Federation to be $7.3 \%$ in 2017 , with half of these individuals (49\%) being younger than 50 years. ${ }^{6}$ The National Diabetes Statistics Report in the USA reported that the total crude prevalence of diabetes was 9.4\% (30.3 million, 2017 US population), with $23.8 \%$ undiagnosed and an additional $33.9 \%$ with pre-diabetes. ${ }^{7}$

In Spain, according to data from the Di@bet.es study, based on OGTT, FPG and HbA1c, $13.8 \%$ of the adult population, adjusted for age and sex, had diabetes, and of these individuals up to $6 \%$ had undiagnosed diabetes. Furthermore, an additional $14.8 \%$ of individuals presented with some type of pre-diabetic state, $3.4 \%$ based on IFG, $9.2 \%$ based on IGT and 2.2\% with disturbances in both, after adjusting for age and sex. ${ }^{8}$ According to the ADA, up to $70 \%$ of people with pre-diabetes will develop overt diabetes throughout their lives. ${ }^{10} 11$ Moreover, each year, $5 \%$ to $10 \%$ of subjects with pre-diabetes will eventually develop overt diabetes, and according to some studies, this percentage can reach up to $18 \%$ per year; however, this rate may vary with the definition of pre-diabetes and population characteristics. ${ }^{12-15}$ It has been shown that over 3 to 5 years, approximately $25 \%$ of subjects progress to T2D, $25 \%$ return to a normal state of glucose tolerance and $50 \%$ remain in the pre-diabetic state. ${ }^{16}$ Thus, the early diagnosis and screening of pre-diabetes are essential steps towards the prevention of its progression or at least the delay of the onset of T2D.

The primary aim of this study was to assess the prevalence of undiagnosed diabetes and pre-diabetes in the healthy population in the Mollerussa cohort. As a secondary objective, we aimed to assess the variables associated with these conditions and to describe the changes in glycaemic status after 1year of follow-up in subjects with pre-diabetes.

\section{METHODS}

\section{Subjects}

This was a prospective population-based cohort study from the semi-rural area of Mollerussa in Catalonia (northeast Spain) selected between March 2011 and July 2014. The description of the cohort and the procedures performed were initially published as a cohort profile. ${ }^{17}$
Briefly, the database of the Catalan Health Institute (ICS) through its Primary Care Electronic Clinical Station (Estació Clínica Electronica d'Atenció Primaria - eCAP) was used to select the population sample. All population is passively included in the Primary Care Electronic Clinical record according to the Spanish health system, which is based on the principles of universality, free access, equity and fairness of financing. ${ }^{18}$ Then, from a total population of 24666 potentially eligible individuals in the healthcare area (subjects older than 25 years and attending any Primary Healthcare Centre in the same health area), 2226 subjects were randomly selected using a randomiser programme (SPSS software V.16.0 for Windows; SPSS), following the principles of simple random sampling, and were then invited to participate by telephone contact. Based on their willingness to join the study, exclusion criteria, consent and baseline laboratory data, 594 subjects aged $\geq 25$ years were finally included. ${ }^{17}$ The exclusion criteria included a previous diagnosis of diabetes (type 1 diabetes, T2D or any specific subtype of diabetes), treatment with oral antidiabetic drugs or the use of metformin for other conditions. In addition, subjects with cardiovascular disease (heart disease, heart failure, aortic stenosis), cancer, kidney disease, anaemia, hepatitis, gastrointestinal diseases, recent abdominal surgery, chronic obstructive pulmonary disease, chronic infectious diseases, use of systemic glucocorticoids or beta blockers or major psychiatric disorders with psychotic symptoms were excluded from the study. Subjects were considered to have hypertension or dyslipidaemia if they were using anti-hypertensive or lipid-lowering agents. Pre-diabetes was defined as any of the following abnormal glycaemic variables: FPG 100 to $<126 \mathrm{mg} / \mathrm{dL}$ or HbAlc 5.7 to $<6.5 \%$; diabetes was defined as FPG $\geq 1256 \mathrm{mg} / \mathrm{dL}$ or HbA1c $\geq 6.5 \%$. Normal glycaemic status was defined by FPG $<100 \mathrm{mg} / \mathrm{dL}$ and HbA1c $<5.7$ according to the 2010 ADA criteria. ${ }^{1}$ Eleven subjects without baseline HbA1c or FPG measurements were excluded. Subjects with prediabetes at baseline $(\mathrm{n}=229)$ underwent a second visit 12 months after the baseline visit, and $166(72.5 \%)$ of them had relevant information at follow-up.

A fasting blood sample was taken to determine glucose, HbAlc, total cholesterol, high density lipoprotein (HDL)-cholesterol, low density lipoprotein (LDL)cholesterol, triglycerides, renal function and other parameters following standard protocols. ${ }^{17}$ The Fatty Liver Index (FLI) was calculated with the equation developed by Bedogni et al. ${ }^{19}$ Insulin resistance was calculated by the homeostatic model assessment- 2 insulin resistance (HOMA2-IR), beta cell function (HOMA2- $\beta$ ) and insulin sensitivity (HOMA2-S) data were calculated with a HOMA2 calculator released by the Diabetes Trials Unit, University of Oxford: HOMA Calculator. This calculator is available at: http:/ /www.dtu.ox.ac.uk/homacalculator/ (updated 11 October 2017). ${ }^{20}$ The estimated glomerular filtration rate (eGFR) was calculated with the Chronic Kidney Disease Epidemiology Collaboration equation. ${ }^{21}$ 


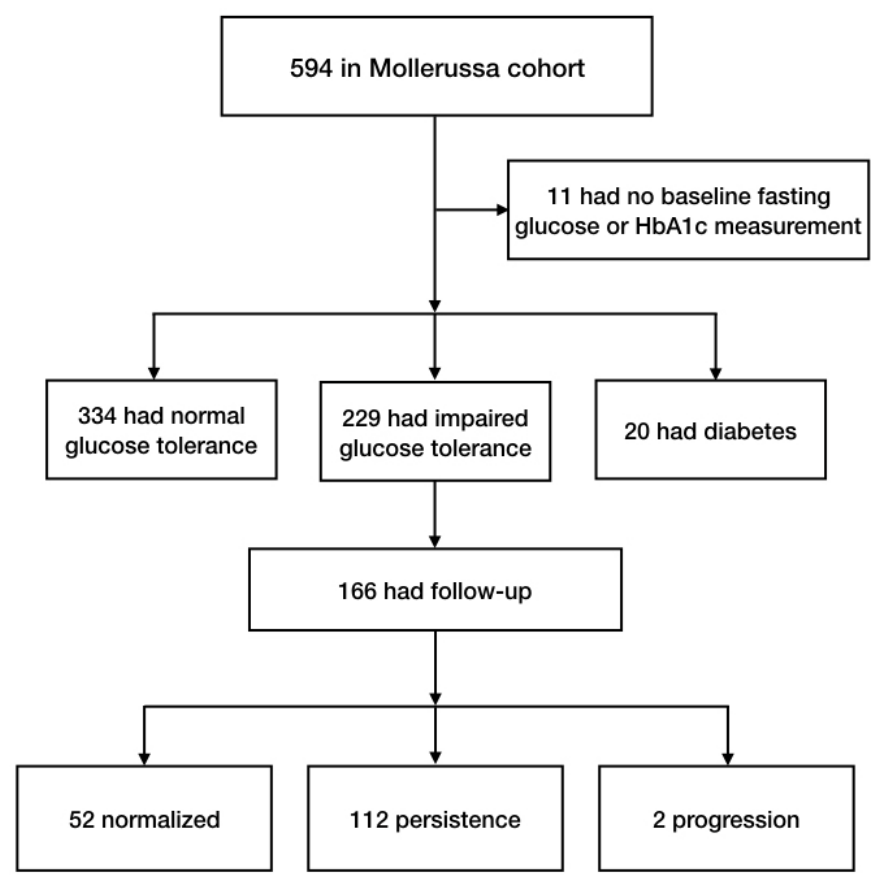

Figure 1 Flow diagram of subjects at baseline and after follow-up. HbA1c,glycated haemoglobin.

Sociodemographic variables were recorded by researchers following a protocol for the inclusion of patients using a standardised baseline questionnaire during the clinical interview. In all cases a physical examination (including weight, height, blood pressure and waist circumference) was carried out by trained research staff. Education level and physical activity were assessed according to the International Standard Classification of Education ${ }^{22}$ and the Spanish-validated International Physical Activity Questionnaire, ${ }^{23}$ respectively. We classified the education level as low level (studied until primary school) and high level (secondary high school education or higher). Physical activity was classified as sedentary or active (not regularly vs regularly active).

The study protocol was conducted following the Declaration of Helsinki. All study participants signed an informed consent form.

\section{Sample size}

The sample size was determined based on an estimated pre-diabetes prevalence of $35.5 \%$ and $38 \%$ using $\mathrm{HbAlc}$ levels and the 2010 ADA criteria, respectively. ${ }^{12425}$ It was estimated that a random sample of 505 subjects was sufficient to assess an estimated prevalence of approximately $30 \%$ with a $95 \% \mathrm{CI}$ and an error of $\pm 4 \% .^{17}$

\section{Statistical methods}

Descriptive statistics of the mean (SD) or median (IQR) were estimated for quantitative variables with a normal or non-normal distribution, respectively. Qualitative variables were assessed using absolute and relative frequencies. Normally distributed data were analysed using the Shapiro-Wilk test. Comparisons between groups of all variables were performed to evaluate the differences.
Student's t-test, analysis of variance, the Mann-Whitney test or the Kruskal-Wallis test were used to assess the differences between groups. The $\mathrm{X}^{2}$ test or Fisher's exact test were used to determine differences in qualitative variables. Tukey's correction was applied to account for multiple tests. Multivariate logistic regression models were used to determine the association of variables with pre-diabetes, isolated FPG, isolated HbA1c and both FPG and $\mathrm{HbAlc}$ at baseline with covariables that were clinically or statistically associated. In the pre-diabetes model, the variables used were age, sex, education level, physical activity, dyslipidaemia, hypertension, family history of diabetes, BMI, waist, glomerular filtration rate and Fatty Liver Index. A stepwise method with selection of variables by backward elimination was used to build the final logistic regression model to predict the normalisation of the glycaemic state; in all models, the goodness-of-fit assumption was tested by the Hosmer-Lemeshow test. The predictive accuracy of the logistic regression model for normalisation was checked by receiver-operating characteristic (ROC) curves and the area under the ROC curve $\left(\mathrm{AUC}_{\mathrm{ROC}}\right)$. ORs with corresponding $95 \%$ CIs are shown, and statistical significance was established as a $p$ value $<0.05$. Data management and all analyses were performed using R statistical software, V.3.3.1, and SPSS software (V.22, IBM, SPSS, Chicago, Illinois, USA).

\section{Patient and public involvement}

This research was done without patient involvement. Patients were not invited to comment on the study design and were not consulted to develop patient-relevant outcomes or interpret the results. Patients were not invited to contribute to the writing or editing of this document for readability or accuracy.

\section{RESULTS}

Out of the 594 individuals recruited, complete data on FPG and HbAlc were available from $583(98.1 \%)$. The prevalence of undiagnosed diabetes was 20 subjects, $3.4 \%$ (95\% CI 2.7 to 4.2 ), and the prevalence of pre-diabetes was 229 subjects, $39.3 \%$ (37.3 to 41.3). Furthermore, the prevalence based on isolated FPG was $7.2 \%$, and that based on isolated HbAlc was $22.8 \%$, while based on the criteria of both FPG and HbA1c, the prevalence was 9.3\% (figure 1).

The differences of clinical and sociodemographic characteristics between normoglycaemic with pre-diabetic and diabetic groups are shown in table 1. Except for sex, family history of diabetes, current smoking status, alcohol consumption status, triglycerides and HDL-cholesterol levels, there were significant differences in the majority of parameters, including age and BMI, between the three groups.

We observed an association in age, BMI, waist circumference, systolic and diastolic blood pressure, alcohol consumption status, hypertension, dyslipidaemia, triglycerides, total cholesterol, LDL-cholesterol, insulin 
Table 1 Clinical and sociodemographic differences among glycaemic status groups of the Mollerussa cohort

\begin{tabular}{|c|c|c|c|c|c|}
\hline & $\begin{array}{l}\text { Normoglycaemia } \\
\text { FPG }<100 \mathrm{mg} / \mathrm{dL} \\
\text { and } \mathrm{HbA1c}<5.7 \%\end{array}$ & $\begin{array}{l}\text { Pre-diabetes } \\
\text { FPG } 100 \text { to } \\
<126 \mathrm{mg} / \mathrm{dL} \text {, or } \\
\text { HbA1c } 5.7 \% \text { to } \\
<6.5 \%\end{array}$ & $\begin{array}{l}\text { Diabetes } \\
\text { FPG } \geq 126 \mathrm{mg} / \mathrm{dL} \text { or } \\
\mathrm{HbA} 1 \mathrm{c} \geq 6.5 \%\end{array}$ & $\begin{array}{l}\text { Mean difference NG vs } \\
\text { PD }(95 \% \mathrm{Cl})\end{array}$ & $\begin{array}{l}\text { Mean difference NG vs } \\
\text { DM }(95 \% \mathrm{Cl})\end{array}$ \\
\hline $\mathrm{N}$ & 334 & 229 & 20 & - & - \\
\hline Sex, women & $193(57.8 \%)$ & 135 (59.0\%) & $13(65.0 \%)$ & $1.2(-7.1$ to 9.4$)$ & $7.2(-14.3$ to 28.8$)$ \\
\hline \multicolumn{6}{|l|}{ BMI categories } \\
\hline Normal weight & $160(50.0 \%)$ & $67(30.2 \%)$ & $4(20.0 \%)$ & $-18.6(-26.6$ to -10.7$)$ & $-27.9(-46.2$ to -9.6$)$ \\
\hline Overweight & $120(37.5 \%)$ & $106(47.7 \%)$ & $5(25.0 \%)$ & 10.4 (2.1 to 18.6$)$ & $-10.9(-30.6$ to 8.7$)$ \\
\hline Obesity & $40(12.5 \%)$ & $49(22.1 \%)$ & $11(55.0 \%)$ & 9.4 (3.1 to 15.8$)$ & 43.0 (20.9 to 65.1$)$ \\
\hline Hypertension & $37(11.1 \%)$ & $49(21.4 \%)$ & $9(45.0 \%)$ & 10.3 (4.0 to 16.6$)$ & 33.9 (11.9 to 55.9 ) \\
\hline Dyslipidaemia & $27(8.08 \%)$ & $39(17.0 \%)$ & $5(25.0 \%)$ & 8.9 (3.2 to 14.6$)$ & $16.9(-2.3$ to 36.1$)$ \\
\hline Family history DM & $94(29.6 \%)$ & $78(37.0 \%)$ & $8(42.1 \%)$ & $5.9(-1.9$ to 13.7$)$ & $11.8(-10.1$ to 33.9$)$ \\
\hline Education, high level & $265(82.6 \%)$ & $145(65.0 \%)$ & $11(55.0 \%)$ & $-16.0(-23.6$ to -8.4$)$ & $-24.3(-46.6$ to -2.1$)$ \\
\hline Physical activity & $243(75.9 \%)$ & $141(63.2 \%)$ & $10(50.0 \%)$ & $-11.2(-19.1$ to -3.3$)$ & $-22.7(-45.2$ to -0.3$)$ \\
\hline Current smoker & $82(24.6 \%)$ & $63(27.5 \%)$ & $3(15.0 \%)$ & $3.0(-4.4$ to 10.4$)$ & $-9.5(-25.9$ to 6.8$)$ \\
\hline Alcohol, g/day & $8.33(13.9)$ & $12.3(21.3)$ & $10.6(17.2)$ & 4.0 (0.9 to 6.9$)$ & $2.2(-5.8$ to 10.3$)$ \\
\hline $\mathrm{FPG}, \mathrm{mg} / \mathrm{dL}$ & $86.6(7.04)$ & $97.0(11.2)$ & $119(15.2)$ & 10.4 (8.8 to 11.9) & 32.6 (28.4 to 36.8$)$ \\
\hline $\mathrm{HbA} 1 \mathrm{c}, \%$ & $5.25(0.26)$ & $5.80(0.29)$ & $6.26(0.54)$ & 0.6 (0.5 to 0.6$)$ & $1.0(0.9$ to 1.1$)$ \\
\hline Insulin, $\mu \mathrm{U} / \mathrm{mL}$ & 7.99 (3.78) & $10.1(5.46)$ & $16.2(17.1)$ & 2.1 (1.2 to 3.0$)$ & $8.2(5.8$ to 10.7$)$ \\
\hline Fatty Liver Index & $34.0(26.9)$ & $44.3(28.6)$ & $59.4(33.0)$ & 10.3 (5.5 to 15.0$)$ & 25.4 (12.8 to 38.0$)$ \\
\hline HOMA2- $\beta$ & $104(31.8)$ & 97.7 (31.9) & $89.0(48.4)$ & $-6.3(-11.8$ to -0.8$)$ & $-14.9(-29.7$ to -0.2$)$ \\
\hline HOMA2-S & $118(52.1)$ & $94.0(43.4)$ & $63.3(26.7)$ & $-23.8(-32.0$ to -15.6$)$ & $-54.4(-76.3$ to -32.6$)$ \\
\hline HOMA2-IR & $1.03(0.48)$ & $1.33(0.72)$ & $2.16(2.08)$ & $0.3(0.2$ to 0.4$)$ & 1.1 (0.8 to 1.4$)$ \\
\hline
\end{tabular}

Mean (SD) and $\mathrm{n}(\%)$.

$\mathrm{BMI}$, body mass index; DBP, diastolic blood pressure; DM, diabetes mellitus; eGFR, estimated glomerular filtration rate; FPG, fasting plasma glucose; $\mathrm{HbA1c}$, glycated haemoglobin; HDL, high-density lipoprotein cholesterol; HOMA2-B, homeostatic model assessment-2 beta cell function; HOMA2IR, homeostatic model assessment-2 insulin resistance; HOMA2-S, homeostatic model assessment-2 insulin sensitivity; LDL, low-density lipoprotein cholesterol; NG, normoglycaemia; PD, pre-diabetes; SBP, systolic blood pressure; T-cholesterol, total cholesterol.

test, FLI and HOMA2-IR, which were higher in individuals with pre-diabetes than in individuals with normoglycaemia and were higher in the diabetic group than in the pre-diabetic group. On the other hand, physical activity, education level, eGFR, HOMA2- $\beta$ and HOMA2-S exhibited a negative trend between the same groups. In the pre-diabetic group, $41.9 \%$ had impaired FPG and $81.7 \%$ had impaired HbA1c. On the other hand, among the newly identified diabetic subjects, up to $80 \%$ met the FPG criteria and $85 \%$ met the HbAlc criteria. The prevalence of pre-diabetes increased with increasing age, with percentages of $17.4 \%, 28.6 \%, 46.4 \%, 50 \%$ and $52.9 \%$ in participants aged $<35$ years, 36 to 45 years, 46 to 55 years, 56 to 65 years and $>65$ years, respectively. Regarding BMI categories of normal weight (BMI $<25 \mathrm{~kg} / \mathrm{m}^{2}$ ), overweight (BMI 25 to $29.9 \mathrm{~kg} / \mathrm{m}^{2}$ ) and obese (BMI $>30 \mathrm{~kg}$ / $\mathrm{m}^{2}$ ), the prevalence of pre-diabetes was $29 \%, 45.9 \%$, and $49 \%$, respectively (online supplementary file 1 figure 1 ).

Table 2 shows the characteristics of pre-diabetic individuals by glycaemic state: isolated FPG, isolated $\mathrm{HbA1c}$ 
Table 2 Clinical and sociodemographic characteristics by glycaemic status of the individuals with pre-diabetes

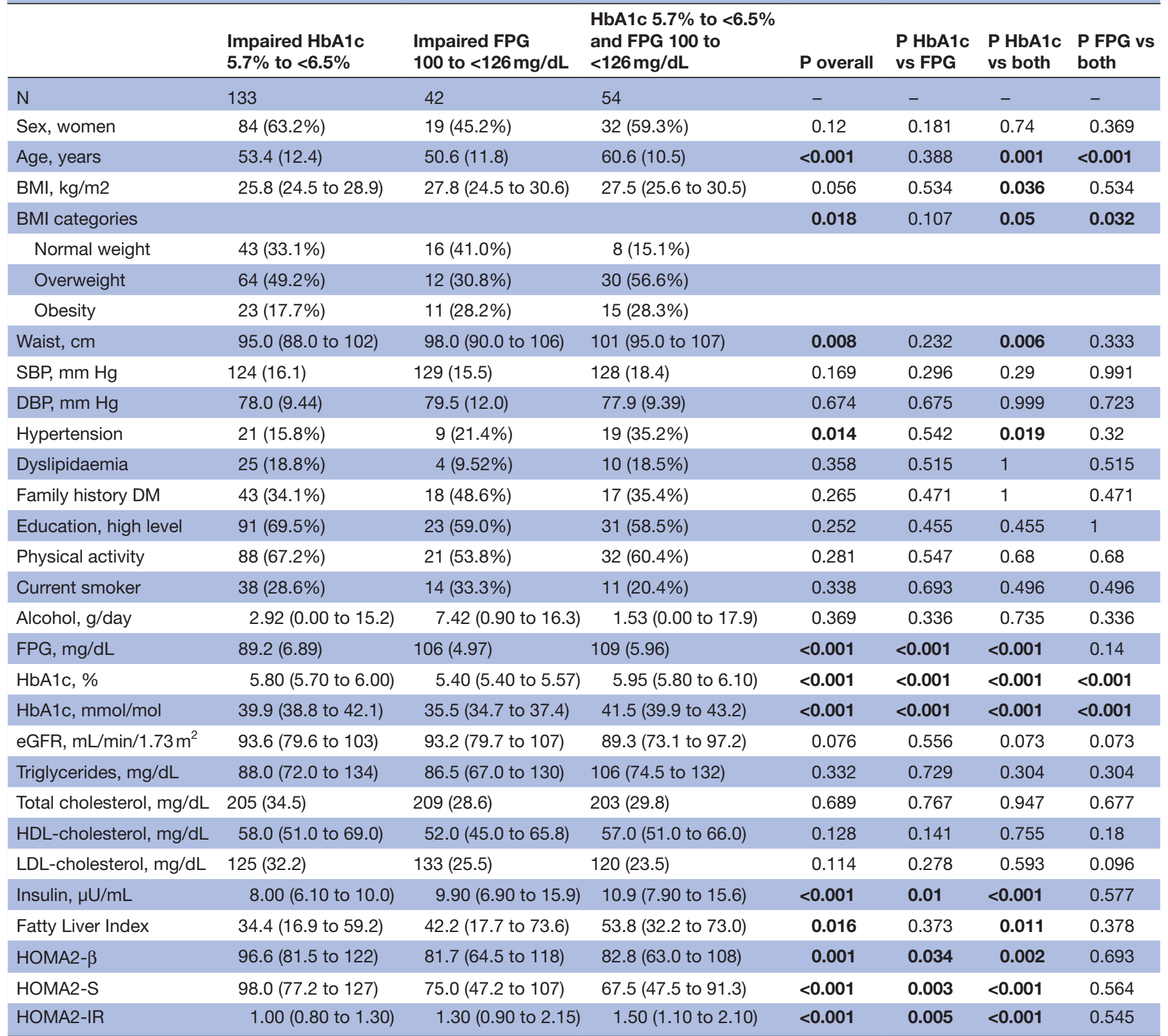

Significant values are shown in bold. Mean (SD), median (IQR) and $\mathrm{n}(\%)$.

$\mathrm{BMI}$, body mass index; DBP, diastolic blood pressure; DM, diabetes mellitus; eGFR, estimated glomerular filtration rate; FPG, fasting plasma glucose; HbA1c, glycated haemoglobin; HDL, high-density lipoprotein; HOMA2- $\beta$, homeostatic model assessment-2 beta cell function; HOMA2-IR, homeostatic model assessment-2 insulin resistance; HOMA2-S, homeostatic model assessment-2 insulin sensitivity; LDL, low-density lipoprotein; SBP, systolic blood pressure.

and both altered FPG and HbAlc. Thus, among the 229 subjects with pre-diabetes, $42(18.3 \%)$ had abnormal isolated FPG, 133 (58.1\%) had abnormal isolated HbA1c and $54(23.6 \%)$ had both abnormal FPG and HbAlc. Patients with both abnormal FPG and HbAlc were older, had larger waist circumferences, had increased FLI and HOMA2-IR, were more likely to be overweight or obese and have hypertension and had lower HOMA2-S. The isolated FPG group had a higher proportion of subjects with a family history of diabetes, higher alcohol consumption, higher levels of total cholesterol and LDL-cholesterol and lower levels of HDL-cholesterol, although none of these differences were statistically significant. Finally, the isolated HbAlc group had an elevated HOMA2- $\beta$. Although there were no statistically significant differences, the proportion of men was higher in the isolated FPG group, whereas the proportion of women was higher in the isolated HbAlc and both FPG and HbAlc groups. Among the three groups, no statistically significant differences were found regarding the following variables: sex, dyslipidaemia, family history of diabetes, education level, physical activity, current smoking status, alcohol consumption, triglycerides, total cholesterol, HDL-cholesterol or LDL-cholesterol. 
Table 3 Outcomes at follow-up of patients with different altered glucose metabolism statuses at baseline

\begin{tabular}{|c|c|c|c|c|c|}
\hline \multirow[b]{2}{*}{ Variables } & \multirow[b]{2}{*}{ Baseline } & \multirow[b]{2}{*}{$\mathrm{N}$ with follow-up } & \multicolumn{3}{|l|}{ Follow-up } \\
\hline & & & Normalised & Persisted & Progressed \\
\hline Pre-diabetes & 229 (39.3\%) & 166 (90.7\%) & 52 (41.6\%) & 112 (57.8\%) & $2(0.6 \%)$ \\
\hline Isolated $\mathrm{HbA} 1 \mathrm{c}$ & $133(22.8 \%)$ & $114(68.7 \%)$ & $47(41.3 \%)$ & $67(58.7 \%)$ & $0(0 \%)$ \\
\hline Both altered & 54 (9.3\%) & 49 (29.5\%) & $4(8.2 \%)$ & $44(89.8 \%)$ & $1(2 \%)$ \\
\hline
\end{tabular}

FPG, fasting plasma glucose; HbA1c, glycated haemoglobin.

\section{Pre-diabetes follow-up}

Of the 229 individuals with pre-diabetes at baseline, 166 (72.5\%) had clinical and laboratory data after 12 months of follow-up. Of them, $52(41.6 \%)$ returned to a normal glycaemic status, $112(57.6 \%)$ persisted in their state of pre-diabetes and only $2(0.6 \%)$ progressed to diabetes. Table 3 shows the outcome of the follow-up of the isolated FPG, HbA1c and both FPG and HbA1c groups.
Association of pre-diabetes with glycaemic status

The multivariate logistic regression model of prediabetes versus normoglycaemia showed that the variables associated with pre-diabetes were older age (OR; $95 \%$ CI) $(1.033 ; 1.011$ to 1.056$)$, higher physical activity levels $(0.546 ; 0.360$ to 0.827$)$, higher BMI $(1.121 ; 1.029$ to 1.222$)$ and a family history of diabetes $(1.543 ; 1.025$ to 2.323) (figure 2A). The models for isolated FPG

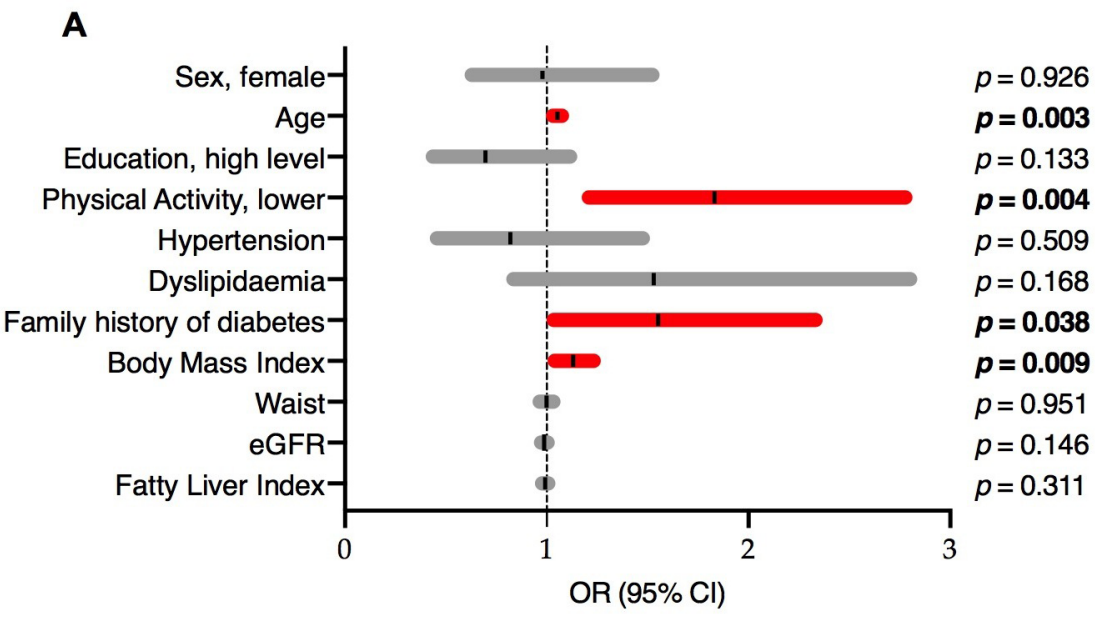

B

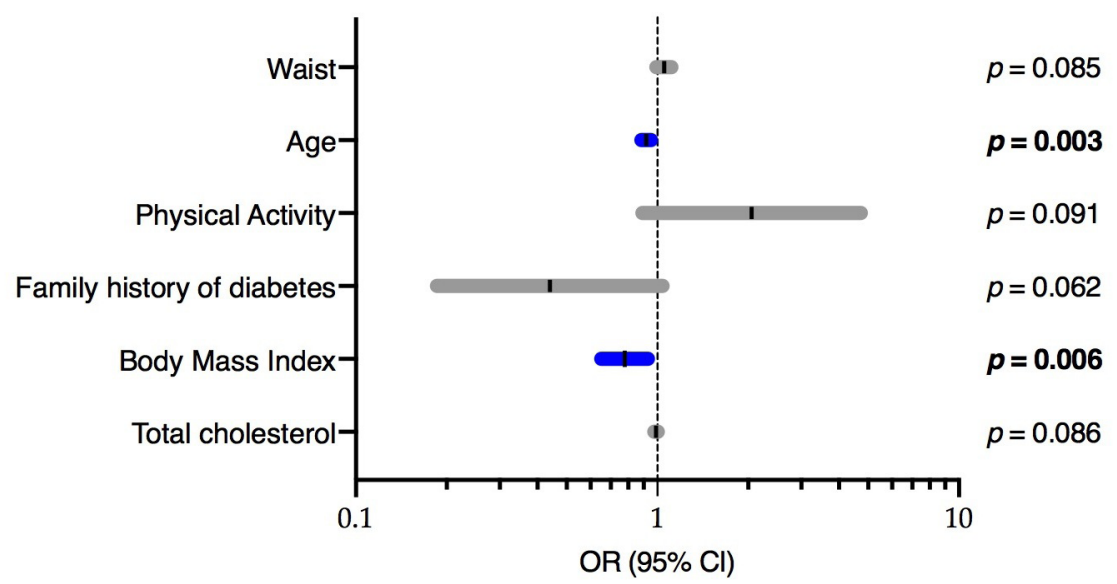

Figure 2 Multivariate logistic regression models: (a) Model of pre-diabetes versus normoglycaemic state in the Mollerussa cohort at baseline. Significant $p$ values are shown in BOLD. Hosmer-Lemeshow test $p=0.295$. (b) Model of normalised versus persisted in subjects with follow-up data. Significant $p$ values are shown in BOLD. Hosmer-Lemeshow test $p=0.931$. eGFR, estimated glomerular filtration rate. 
alterations, isolated HbA1c alterations and both FPG and HbA1c alterations are shown in online supplementary file 2 tables 1, 2 and 3, respectively. The variables associated with isolated FPG were older age (1.032; 1.008 to 1.057 ), higher physical activity levels $(0.535 ; 0.318$ to 0.899$)$ and a family history of diabetes $(1.798 ; 1.067$ to 3.028$)$. On the other hand, the only variable associated with impaired HbA1c was older age $(1.048 ; 1.029$ to 1.067$)$. Finally, in the model for altered FPG and $\mathrm{HbAlc}$, the variables associated were older age $(1.056 ; 1.026$ to 1.086$)$ and high FLI (1.031; 1.002 to 1.061$)$.

\section{Prediction of normalisation}

Logistic regression model, as described in the methods section, starting with the variables age, sex, waist circumference, BMI, hypertension, physical activity, family history of diabetes, education level, total cholesterol, HDL-cholesterol, FLI and HOMA2-IR, was performed to identify factors independently associated with the prediction of glycaemic status normalisation (online supplementary file 2 table 4$)$. The variables that predicted glycaemic normalisation were older age $(0.948 ; 0.916$ to 0.982$)$ and BMI (0.779; 0.651 to 0.931) (figure 2B); this model had a good predictive ability $\left(\mathrm{AUC}_{\mathrm{ROC}} 0.77 ; \mathrm{p}<0.001\right)$ (online supplementary file 3 figure 2 ).

\section{DISCUSSION}

We found that the prevalence of undiagnosed diabetes was $3.4 \%$, and the prevalence of pre-diabetes was $39.3 \%$ in this semi-rural population in Catalonia (northeast Spain). The prevalence of pre-diabetes was three-fold higher based on HbAlc than that based on FPG. Subjects with pre-diabetes defined by both HbAlc and FPG criteria had unfavourable clinical and sociodemographic profiles related to increased cardiovascular risk. These factors were older age; abdominal obesity; higher triglycerides; increased FLI and a higher proportion of overweight, obesity and hypertension. In our population, age was the variable most strongly associated with pre-diabetes based on all specific glycaemic status variables: isolated impaired FPG, isolated impaired HbAlc or both impaired FPG and HbA1c. Other variables associated with pre-diabetes were lower physical activity levels, a family history of diabetes and obesity. Finally, the characteristics related to normalisation at follow-up were younger age and lower BMI.

The prevalence of pre-diabetes and undiagnosed diabetes in our healthy population were within the ranges found in other population studies defining pre-diabetes based on the 2010 ADA criteria, using FPG and/or HbA1c. Among these studies, a large national Chinese study (with 170287 subjects) showed a prevalence of pre-diabetes of $35.7 \%$ and a prevalence of undiagnosed diabetes of $6.9 \% .^{26}$ In a study of the Caribbean population, the corresponding figures were $44.1 \%$ for pre-diabetes and $7.3 \%$ for undiagnosed diabetes. $^{27}$ In England, based on HbAlc levels, the prediabetes prevalence was $35.5 \%$ in the adult population in 2011. ${ }^{24}$ In these studies, the prevalence of pre-diabetes was higher in older, overweight and obese participants. ${ }^{24} 2627$ Many other studies found this relationship of age and obesity with the risk and incidence of diabetes. ${ }^{28-31}$

In the 1999 to 2002 National Health and Nutrition Examination Survey (NHANES), the prevalence of undiagnosed diabetes was $2.8 \%$, and up to $26 \%$ of the participants had IFG. ${ }^{32}$ However, the age-standardised prevalence of prediabetes based on HbA1c and FPG combined was similar in the periods between 1999 and 2002 and 2003 and 2006 at $29.2 \%$ and $29.3 \%$, respectively, but increased significantly to $36.2 \%$ in the period between 2007 and $2010 .^{33}$ This prevalence continued to increase to as high as $38 \%$ in 2012 among adults from the USA. ${ }^{25}$ The change in the prevalence of pre-diabetes over time occurred because of a significant change in elevated $\mathrm{HbA1c}$, whereas the prevalence based on elevated FPG was similar over this period. ${ }^{33}$ Thus, in our population, as in the NHANES study, HbA1c was the most significant contributor to pre-diabetes prevalence, followed by FPG, which is in concordance with the findings in the Caribbean population ${ }^{27}$ and discordant with the reports from the NHANES study between 2011 and 2014 in which they reported that FPG was the most significant contributor to pre-diabetes prevalence followed by HbAlc. ${ }^{34}$ Our results show that individuals with isolated impaired HbAlc when diagnosed with pre-diabetes might have a slightly better cardiometabolic risk profile than those with isolated FPG, while those individuals with both impaired FPG and HbAlc had the worst cardiovascular risk. These results are in line with the findings of the prospective observational study in the primary care setting of a Spanish cohort with pre-diabetes (PREDAPS) of our group. $^{35} 36$

Additionally, two meta-analyses found that among individuals with pre-diabetes based on the ADA criteria, allcause and cardiovascular disease (CVD) mortality were increased $^{37}$ and that the risk of cardiovascular disease increased independently of the glucose assessment in comparison to the risk of normoglycaemic subjects. ${ }^{38}$ Moreover, a recent study concluded that those who returned to normoglycaemia from FPG-defined or HbAlc-defined pre-diabetes were not at reduced risk of future CVD or death. ${ }^{39}$ Studies of shorter duration, over 3 to 5 years, have shown that approximately $25 \%$ of subjects progress to diabetes, $25 \%$ return to a normal state of glucose tolerance and $50 \%$ remain in the pre-diabetic state; ${ }^{16}$ after 1 year, $18.8 \%$ of subjects with pre-diabetes returned to normoglycaemia and approximately $30 \%$ with abnormal FPG, 29.1\% with abnormal HbA1c and $7.6 \%$ with abnormalities in both FPG and HbA1c returned to a normal state of glucose tolerance. ${ }^{40}$ In our findings from a 1-year follow-up, the rate of reversion from prediabetes to normoglycaemia was approximately $40 \%$, and approximately $60 \%$ of participants remained in the prediabetic state. On the other hand, lifestyle modifications, such as weight loss and increased physical activity, among other factors associated with pre-diabetes, reduced the risk of diabetes among these subjects. ${ }^{13} 41$ According to these reports, in our study, lower BMI was a factor that was 
independently associated with the normalisation of the glycaemic state, and an active lifestyle decreased the risk of having pre-diabetes.

The results of this study need to be interpreted in light of its strengths and weaknesses. First, the number of participants in our study is smaller in comparison to other studies. In addition, the study may not be representative of urban areas in our region. Thus, the results may not be generalisable to other territories with different population characteristics in our country. However, the Mollerussa cohort is a representative sample of the region, which is a specific semi-rural area that has never been specifically investigated. Second, our study sample is probably healthier than the general population, as we excluded subjects with already known diabetes and other comorbidities, a lower number of subjects were counted in the denominator, thus resulting in a higher prevalence of this condition. Third, we did not assess glucose tolerance through an oral glucose tolerance test, which is common in most population studies. Although this assay is sensitive, it is also less specific for identifying subjects who could develop diabetes. ${ }^{42}$ Furthermore, the oral glucose tolerance test has a low reproducibility and is a rather time-consuming and expensive procedure. ${ }^{93}$ Conversely, HbAlc and FPG are cost-effective and more convenient for patients. Currently, FPG is an accepted screening method to detect diabetes and pre-diabetes. HbA1c improves the sensitivity of FPG in the detection of early T2D in high-risk subjects ${ }^{324}$ and is a better predictor of $\mathrm{CV}$ events than FPG. ${ }^{45}$ Fourth, we only followed up those participants with pre-diabetes. Thus, we could not analyse the probability of changing from normoglycaemia to pre-diabetes or diabetes in this study. Finally, it is probable that the use of the WHO pre-diabetes criteria in our study would have resulted in a smaller proportion of subjects who returned to a normal glycaemic state. The WHO established a normal concentration of FPG between 110 and $<126 \mathrm{mg} / \mathrm{dL}^{46}$

\section{Conclusions}

For the first time, our study provides information on the prevalence of diabetes and pre-diabetes in the Mollerussa healthcare area, a Mediterranean semi-rural area in northeast Spain. Individuals with pre-diabetes had a more unfavourable cardiometabolic risk profile than normoglycaemic subjects. Moreover, individuals with abnormalities in both criteria used to diagnose pre-diabetes had the worst risk profile. Finally, after 1 year of follow-up, few people progressed to diabetes, while more than $40 \%$ returned to a normal glycaemic state and nearly $60 \%$ persisted in the pre-diabetic state. These results suggest that the use of both FPG and HbAlc criteria in clinical practice could help identify people with high diabetes and cardiovascular risk. Moreover, the identification of individuals with pre-diabetes provides an opportunity for intervention through lifestyle modification and pharmacological treatments not only to reduce the development of diabetes.

\section{Author affiliations}

${ }^{1}$ Primary Health Care Centre Cervera, Gerència d'Atenció Primaria, Institut Català de la Salut, Lleida, Spain

${ }^{2}$ Department of Medicine, University of Lleida \& Biomedical Research Institute of Lleida, Lleida, Spain

${ }^{3}$ Primary Health Care Centre Igualada Nord, Gerència d'Atenció Primaria, Institut Català de la Salut, Lleida, Spain

${ }^{4}$ Department of Nutrition and Dietetics, Avantmedic, Lleida, Spain

${ }^{5}$ Department of Endocrinology \& Nutrition, University Hospital Germans Trias I Pujol \& Health Sciences Research Institute, Badalona, Spain

${ }^{6}$ Unitat de Suport a la Recerca Lleida, Fundació Institut Universitari per a la Recerca a I'Atenció Primària de Salut Jordi Gol i Gurina (IDIAPJGol), \& CIBER of Epidemiology and Public Health (CIBERESP), Lleida, Spain

${ }^{7}$ Department of Cardiovascular, Vall d'Hebron Research Institute, Barcelona, Spain

${ }^{8}$ Primary Health Care Centre Tàrrega, Gerència d'Atenció Primaria, Institut Català de la Salut, Lleida, Spain

${ }^{9}$ Primary Health Care Centre Mollerussa, Gerència d'Atenció Primaria, Institut Català de la Salut, Lleida, Spain

${ }^{10}$ Primary Health Care Centre Guissona, Gerència d'Atenció Primaria, Institut Català de la Salut, Lleida, Spain

${ }^{11}$ DAP-Cat Group, Unitat de Suport a la Recerca Barcelona, Fundació Institut Universitari per a la Recerca a l'Atenció Primària de Salut Jordi Gol i Gurina (IDIAPJGol), \& CIBER on Diabetes and Associated Metabolic Diseases (CIBERDEM), Barcelona, Spain

${ }^{12}$ Primary Health Care Centre Raval Sud, Gerència d'Atenció Primaria Barcelona, Institut Català de la Salut, Barcelona, Spain

${ }^{13}$ Primary Health Care Centre La Mina, Gerència d'Atenció Primària Barcelona, Institut Català de la Salut, Barcelona, Spain

${ }^{14}$ DAP-Cat Group, Unitat de Suport a la Recerca Barcelona, Fundació Institut Universitari per a la Recerca a l'Atenció Primària de Salut Jordi Gol i Gurina (IDIAPJGol), Barcelona, Spain

${ }^{15}$ Department of Endocrinology \& Nutrition, Hospital de la Santa Creu i Sant Pau \& Institut d'Investigació Biomédica Sant Pau (IIB Sant Pau), \& CIBER on Diabetes and Associated Metabolic Diseases (CIBERDEM), Barcelona, Spain

Acknowledgements The authors thank Jordi Real for their valuable assistance in conducting the statistical analysis. CIBER of Diabetes and Associated Metabolic Diseases is an initiative from Instituto de Salud Carlos III (Plan Nacional de I+D+I and Fondo Europeo de Desarrollo Regional).

Contributors MF, EC and DM conceived and designed the study; MBV, JFN and MMC participated in the study design; MBV, MF, NA, MGC, NM, AM and CC collected the data; EC and JRM performed the statistical analyses; MF, EC, MMC and DM wrote the manuscript. All authors critically reviewed the manuscript and approved the final version to be published.

Funding This study was funded by Institut Universitari d'Investigació en Atenció Primària Jordi Gol (IDIAP Jordi Gol).

Competing interests None declared.

Patient consent for publication Not required.

Ethics approval The project was approved by the Ethics Committee of the Primary Health Care University Research Institute (IDIAP) Jordi Gol (PI12/043) Barcelona, Spain.

Provenance and peer review Not commissioned; externally peer reviewed.

Data availability statement Data are available upon reasonable request. Readers may contact Dr Didac Mauricio (didacmauricio@gmail.com) regarding the data.

Open access This is an open access article distributed in accordance with the Creative Commons Attribution Non Commercial (CC BY-NC 4.0) license, which permits others to distribute, remix, adapt, build upon this work noncommercially, and license their derivative works on different terms, provided the original work is properly cited, appropriate credit is given, any changes made indicated, and the use is non-commercial. See: http://creativecommons.org/ licenses/by-nc/4.0/.

\section{ORCID iDs}

Manel Mata-Cases http://orcid.org/0000-0003-3693-3622

Esmeralda Castelblanco http://orcid.org/0000-0002-2061-6270

Didac Mauricio http://orcid.org/0000-0002-2868-0250 


\section{REFERENCES}

1 Olson DE, Rhee MK, Herrick K, et al. Screening for diabetes and pre-diabetes with proposed A1C-based diagnostic criteria. Diabetes Care 2010;33:2184-9.

2 American Diabetes Association. 2. classification and diagnosis of diabetes. Diabetes Care 2017;40:S11-24.

3 Brannick B, Wynn A, Dagogo-Jack S. Prediabetes as a toxic environment for the initiation of microvascular and macrovascular complications. Exp Biol Med 2016;241:1323-31.

4 Ferrannini E. Definition of intervention points in prediabetes. Lancet Diabetes Endocrinol 2014;2:667-75.

5 American Diabetes Association. 2. Classification and Diagnosis of Diabetes: Standards of Medical Care in Diabetes-2019. Diabetes Care 2019;42:S13-28.

6 International diabetes Federation diabetes atlas. 8th ED, 2017. Available: http://www.diabetesatlas.org/ [Accessed Jun 2019].

7 Centers for Disease Control and Prevention. National diabetes statistics report, 2017. Atlanta, GA Centers for disease control and prevention, US. Dept of Health and Human Services; 2017. https:// www.cdc.gov/diabetes/data/statistics/statistics-report.htm

8 Soriguer F, Goday A, Bosch-Comas A, et al. Prevalence of diabetes mellitus and impaired glucose regulation in Spain: the Di@bet.es Study. Diabetologia 2012;55:88-93.

9 Mata-Cases M, Artola S, Escalada J, et al. Consenso sobre La detección Y El manejo de la prediabetes. Grupo de Trabajo de Consensos Y Guías Clínicas de la Sociedad Española de diabetes. Revista Clínica Española 2015;215:117-29.

10 Nathan DM, Davidson MB, DeFronzo RA, et al. Impaired fasting glucose and impaired glucose tolerance: implications for care. Diabetes Care 2007;30:753-9.

11 Tabák AG, Herder C, Rathmann W, et al. Prediabetes: a high-risk state for diabetes development. The Lancet 2012;379:2279-90.

12 Tuomilehto J, Lindström J, Eriksson JG, et al. Prevention of type 2 diabetes mellitus by changes in lifestyle among subjects with impaired glucose tolerance. N Engl J Med 2001;344:1343-50.

13 Knowler WC, Barrett-Connor E, Fowler SE, et al. Reduction in the incidence of type 2 diabetes with lifestyle intervention or metformin. N Engl J Med 2002;346:393-403.

14 Ramachandran A, Snehalatha C, Mary S, et al. The Indian diabetes prevention programme shows that lifestyle modification and metformin prevent type 2 diabetes in Asian Indian subjects with impaired glucose tolerance (IDPP-1). Diabetologia 2006;49:289-97.

15 Gerstein HC, Santaguida P, Raina P, et al. Annual incidence and relative risk of diabetes in people with various categories of dysglycemia: a systematic overview and meta-analysis of prospective studies. Diabetes Res Clin Pract 2007;78:305-12.

16 Paulweber B, Valensi P, Lindström J, et al. A European evidencebased guideline for the prevention of type 2 diabetes. Horm Metab Res 2010;42 Suppl 1:S3-36.

17 Vilanova MB, Falguera M, Marsal JR, et al. Prevalence, clinical features and risk assessment of pre-diabetes in Spain: the prospective Mollerussa cohort study. BMJ Open 2017;7:e015158-8.

18 Bernal-Delgado E, García-Armesto S, Oliva J, et al. Spain: health system review. Health Syst Transit 2018;20:1-179.

19 Bedogni G, Bellentani S, Miglioli L, et al. The fatty liver index: a simple and accurate predictor of hepatic steatosis in the general population. BMC Gastroenterol 2006;6:44-7.

20 Levy JC, Matthews DR, Hermans MP. Correct homeostasis model assessment (HOMA) evaluation uses the computer program. Diabetes Care 1998;21:2191-2.

21 Levey AS, Stevens LA, Schmid CH, et al. A new equation to estimate glomerular filtration rate. Ann Intern Med 2009;150:604-12.

22 OECD/Eurostat/UNESCO Institute for Statistics. ISCED 2011 operational manual: guidelines for classifying national education programmes and related qualifications. Paris: OECD Publishing, 2015.

23 Roman-Viñas B, Serra-Majem L, Hagströmer M, et al. International physical activity questionnaire: reliability and validity in a Spanish population. Eur J Sport Sci 2010;10:297-304.

24 Mainous AG, Tanner RJ, Baker R, et al. Prevalence of prediabetes in England from 2003 to 2011: population-based, cross-sectional study. BMJ Open 2014;4:e005002-8.
25 Menke A, Casagrande S, Geiss L, et al. Prevalence of and trends in diabetes among adults in the United States, 1988-2012. JAMA 2015;314:1021-9.

26 Wang L, Gao P, Zhang M, et al. Prevalence and ethnic pattern of diabetes and prediabetes in China in 2013. JAMA 2017;317:2515-6.

27 Unwin N, Howitt C, Rose AMC, et al. Prevalence and phenotype of diabetes and prediabetes using fasting glucose vs $\mathrm{HbA} 1 \mathrm{c}$ in a Caribbean population. J Glob Health 2017;7:1-11.

28 Soriguer F, Rojo-Martínez G, Almaraz MC, et al. Incidence of type 2 diabetes in southern Spain (Pizarra study). Eur J Clin Invest 2008;38:126-33.

29 DECODE Study Group. Age- and sex-specific prevalences of diabetes and impaired glucose regulation in 13 European cohorts. Diabetes Care 2003;26:61-9.

30 Edelstein SL, Knowler WC, Bain RP, et al. Predictors of progression from impaired glucose tolerance to NIDDM: an analysis of six prospective studies. Diabetes 1997;46:701-10.

31 Burke JP, Williams K, Gaskill SP, et al. Rapid rise in the incidence of type 2 diabetes from 1987 to 1996. Arch Intern Med 1999;159:1450-6.

32 Cowie CC, Rust KF, Byrd-Holt DD, et al. Prevalence of diabetes and impaired fasting glucose in adults in the U.S. population: National health and nutrition examination survey 1999-2002. Diabetes Care 2006;29:1263-8.

33 Bullard KM, Saydah SH, Imperatore G, et al. Secular changes in U.S. prediabetes prevalence defined by hemoglobin A1c and fasting plasma glucose: National health and nutrition examination surveys, 1999-2010. Diabetes Care 2013;36:2286-93.

34 Menke A, Casagrande S, Cowie CC. Contributions of A1c, fasting plasma glucose, and 2-hour plasma glucose to prediabetes prevalence: NHANES 2011-2014. Ann Epidemiol 2018;28:681-5.

35 Giráldez-García C, Sangrós FJ, Díaz-Redondo A, et al. Cardiometabolic Risk Profiles in Patients With Impaired Fasting Glucose and/or Hemoglobin A1c 5.7\% to 6.4\%. Medicine 2015;94:e1935-8.

36 Franch-Nadal J, Caballería L, Mata-Cases M, et al. Fatty liver index is a predictor of incident diabetes in patients with prediabetes: the PREDAPS study. PLoS One 2018;13:e0198327-17.

37 Huang Y, Cai X, Mai W, et al. Association between prediabetes and risk of cardiovascular disease and all cause mortality: systematic review and meta-analysis. BMJ 2016;355:i5953.

38 Levitan EB, Song Y, Ford ES, et al. Is nondiabetic hyperglycemia a risk factor for cardiovascular disease? Arch Intern Med 2004;164:2147-55

39 Vistisen D, Kivimäki M, Perreault L, et al. Reversion from prediabetes to normoglycaemia and risk of cardiovascular disease and mortality: the Whitehall II cohort study. Diabetologia 2019:62:1385-90.

40 Giráldez-García C, García Soidán FJ, Serrano Martín R, et al. Evolución de pacientes Con prediabetes en Atención Primaria de Salud (PREDAPS): resultados del primer año de seguimiento. Diabetes practica 2014;5:1-48.

41 Díaz-Redondo A, Giráldez-García C, Carrillo L, et al. Modifiable risk factors associated with prediabetes in men and women: a crosssectional analysis of the cohort study in primary health care on the evolution of patients with prediabetes (PREDAPS-Study). BMC Fam Pract 2015;16.

42 Unwin N, Shaw J, Zimmet P, et al. Impaired glucose tolerance and impaired fasting glycaemia: the current status on definition and intervention. Diabet Med 2002;19:708-23.

43 Gossain VV, Aldasouqi S. The challenge of undiagnosed prediabetes, diabetes and associated cardiovascular disease. Int $J$ Diabetes Mellit 2010;2:43-6.

44 Droumaguet C, Balkau B, Simon D, et al. Use of $\mathrm{HbA} 1 \mathrm{c}$ in predicting progression to diabetes in French men and women: data from an epidemiological study on the insulin resistance syndrome (DESIR). Diabetes Care 2006;29:1619-25.

45 Selvin E, Steffes MW, Zhu H, et al. Glycated hemoglobin, diabetes, and cardiovascular risk in nondiabetic adults. $N$ Engl J Med 2010;362:800-11.

46 World Health Organization. Use of glycated haemoglobin (HbA1c) in the diagnosis of diabetes mellitus. Ginebra: World Health Organization, 2011. 